
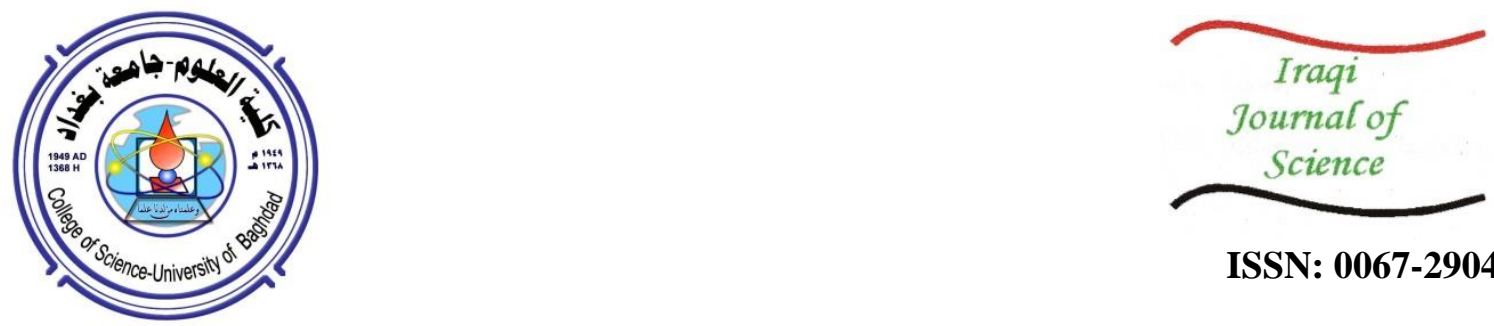

ISSN: 0067-2904

\title{
Heat Transfer Analysis and Magnetohydrodynamics Effect on Peristaltic Transport of Ree-Eyring Fluid in Rotating Frame
}

\author{
Batool A. Almusawi*, Ahmed M. Abdulhadi \\ Department of Mathematics, College of science, University of Baghdad, Baghdad, Iraq \\ Received: 16/2/2021 Accepted: 16/5/2021

\begin{abstract}
This paper discusses Ree-Eyring fluid's peristaltic transport in a rotating frame and examines the impacts of Magnetohydrodynamics (MHD). The results deal with systematically (analytically) applying each of the governing equations of Ree-Eyring fluid, the axial and secondary velocities, flow rate due to auxiliary stream, and bolus. The effects of some distinctive variables, such as Hartman number, heat source/sink, and amplitude ratio, are taken under consideration and illustrated through graphs.
\end{abstract}

Keywords: Rotating frame, Ree-Eyring fluid, Non-Newtonian fluid, Heat transfer, Magnetic field.

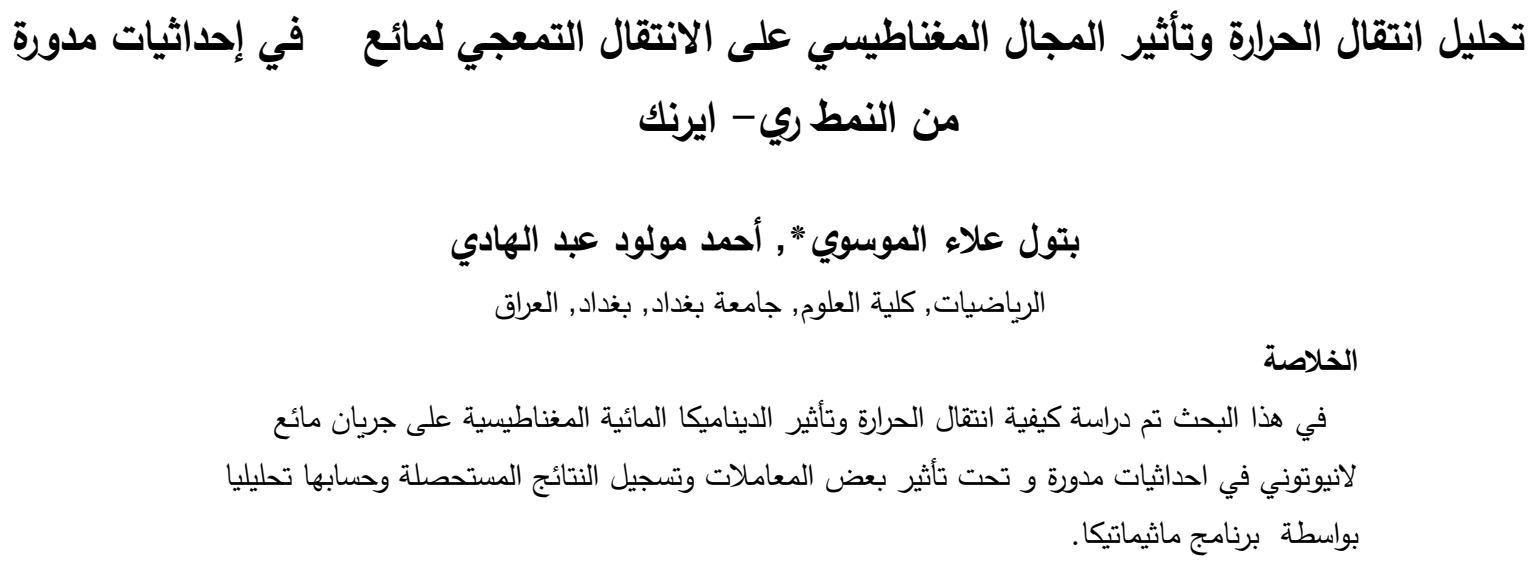

\section{Introduction}

A non-Newtonian fluid is a fluid that is not subject to Newton's laws of viscosity. It has become important in many researches. The viscosity (the gradual deformation by shear or tensile stresses) of non-Newtonian fluids is dependent on shear rate or shear rate history. In a Newtonian fluid, the relation between the shear stress and the shear rate is linear but, in a non-Newtonian fluid, this relation is different. For example, many salt solutions and molten polymers are non-Newtonian fluids. Here we studied the Ree-Eyring model $[1,2]$ because it is one of the important fluids that can be converted into a Newtonian fluid model for high and low shear rates. Ellahi et al. [3] studied the mathematical analysis of peristaltic transport of an Eyring- Powell fluid through a porous rectangular duct.

MHD is the study of the behavior of electrically conductive fluids when exposed to a magnetic field, that is, the study of magnetic properties and the behavior of those fluids along with the changes that occur. Examples of this fluid include plasma, liquid minerals, and others. The set of equations describing MHD is a combination of the Navier-Stokes equations of fluid dynamics and Maxwell's equations of electromagnetism. Solving these equations is achieved either analytically or numerically.

*Email: Batool.Hussien1203@sc.uobaghdad.edu.iq 
The rotation phenomenon has vast applications in cosmic and geophysical flows and helps to better comprehend galaxy formation and ocean circulation. Rotational diffusion accounts for nanoparticle orientation in fluids. The following is a review of studies that discuss the effects of rotation. Hayat and Zahir [4] presented heat transfer analysis on peristaltic transport of Ree- Eyring fluid in rotating frame. Safa and Abdulhadi [5] performed unsteady heat transfer analysis on MHD flow of a second grade fluid in a channel with porous medium. Farah and Abdulhadi [6] analyzed the effect of inclined magnetic field on peristaltic flow of Bingham plastic fluid in an inclined symmetric channel with slip conditions. Khan et al. [7] studied the entropy generation in radiative flow of Ree-Eyring fluid due to due rotating disks. Wissam and Dheia [8] presented the influence of heat transfer on magneto hydrodynamics oscillatory flow for Williamson fluid through a porous medium. There have also been other attempts to study MHD [9-11].

The aim of the present investigation is to examine the peristaltic transport of Ree-Eyring liquid in a rotating frame, taking into consideration the convective condition. It is worth specifying that such examination for Ree-Eyring liquid is critical for different applications in biomedicine and designing. For further clarification, this article is organized into Section 2 that presents the physical modeling statement for our problem. The axial velocity, secondary velocity, temperature, and stream function are interpreted for relevant parameters and analyzed through graphs in Section 4. Finally, conclusions are given in Section 5.

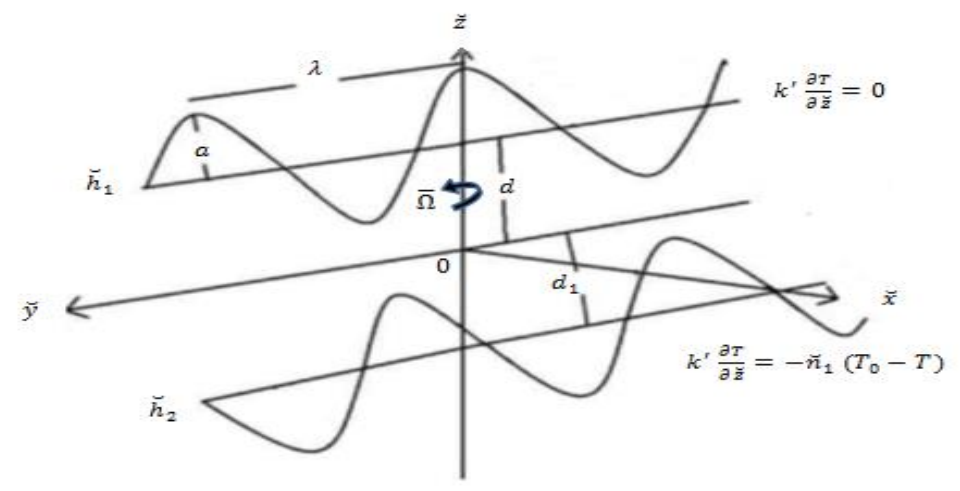

Figure -1 Geometry graph of the flow.

\section{Mathematical formulation}

Let us consider an incompressible Ree-Eyring fluid material through a channel of width $\left(d+d_{1}\right)$. The fluid is electrically conducting by an external magnetic field, $\mathrm{B}=\left(0,0, \beta_{0}\right)$, that is applied to it. Both the channel and fluid rotate with a uniform angular velocity $\bar{\Omega}$ about the $\breve{z}$-axis, as shown in Figure-1. Mathematical expression for wave propagation along the walls of the channel at $\breve{z}=\mp \breve{h}_{1,2}$ can be written as:

$$
\begin{gathered}
\breve{z}=\breve{h}_{1}(\breve{x}, \breve{t})=d+m^{\prime} \breve{x}+a \sin \left[\frac{2 \pi}{\lambda}(\breve{x}-c \breve{t})\right] \\
\breve{z}=\breve{h}_{2}(\breve{x}, \breve{t})=-d_{1}-m^{\prime} \breve{x}-b\left[\sin \frac{2 \pi}{\lambda}(\breve{x}-c \breve{t})+\phi^{\prime}\right]
\end{gathered}
$$

in which $\breve{h}_{1}, \breve{h}_{2}$ are the upper and lower wall, respectively, $a, b$ are the amplitudes of the waves along the lower and upper walls, respectively, $\phi^{\prime}$ the phase difference, and $m^{\prime}$ is the non- uniform parameter.

The governing equations of continuity and energy and the momentum equation for incompressible, irrotational, laminar flow can be written as [12]

$$
\begin{gathered}
\frac{\partial \breve{u}}{\partial \breve{x}}+\frac{\partial \breve{w}}{\partial \breve{z}}=0 \\
\rho \frac{d \breve{u}}{d \breve{t}}-2 \rho \bar{\Omega} \breve{v}=-\frac{\partial \breve{P}}{\partial \breve{x}}+\frac{\partial \breve{S}_{\breve{x} \breve{x}}}{\partial \breve{x}}+\frac{\partial \breve{S}_{\breve{x} \breve{y}}}{\partial \breve{y}}+\frac{\partial \breve{S}_{\breve{x} \breve{z}}}{\partial \breve{z}}-\sigma \beta_{0}^{2} \breve{u} \\
\rho \frac{d \breve{v}}{d \breve{t}}+2 \rho \bar{\Omega} \breve{u}=-\frac{\partial \breve{P}}{\partial \breve{y}}+\frac{\partial \breve{S}_{\breve{y} \breve{x}}}{\partial \breve{x}}+\frac{\partial \breve{S}_{\breve{y} \breve{y}}}{\partial \breve{y}}+\frac{\partial \breve{S}_{\breve{y} \breve{z}}}{\partial \breve{z}}
\end{gathered}
$$




$$
\begin{gathered}
\rho \frac{d \breve{w}}{d \breve{t}}=-\frac{\partial \breve{P}}{\partial \breve{z}}+\frac{\partial \breve{S}_{\breve{z} \breve{x}}}{\partial \breve{x}}+\frac{\partial \breve{S}_{\breve{z} \breve{y}}}{\partial \breve{y}}+\frac{\partial \breve{S}_{\breve{z} \breve{z}}}{\partial \breve{z}} \\
\rho c_{p} \frac{d T}{d \breve{t}}=k^{\prime}\left[\frac{\partial^{2} T}{\partial \breve{x}^{2}}+\frac{\partial^{2} T}{\partial \breve{y}^{2}}+\frac{\partial^{2} T}{\partial \breve{z}^{2}}\right]+\breve{S}_{\breve{x} \breve{x}} \frac{\partial \breve{u}}{\partial \breve{x}}+\breve{S}_{\breve{x} \breve{z}}\left(\frac{\partial \breve{u}}{\partial \breve{z}}+\frac{\partial \breve{w}}{\partial \breve{x}}\right)+\breve{S}_{\breve{z} \breve{z}} \frac{\partial \breve{w}}{\partial \breve{z}}+Q_{0}\left(T-T_{0}\right)
\end{gathered}
$$

where $\lambda, c, \breve{t}, \rho, c_{p}, k^{\prime}, Q_{0}, \breve{P}, \sigma$, and $\frac{d}{d \breve{t}}$ represent the wavelength, constant speed, time of the wave, fluid density, specific heat, thermal conductivity, heat source/sink, modified pressure, electric conductivity, and material time derivative, respectively.

The stress tensor of Ree-Eyring fluid model is defined as [13]

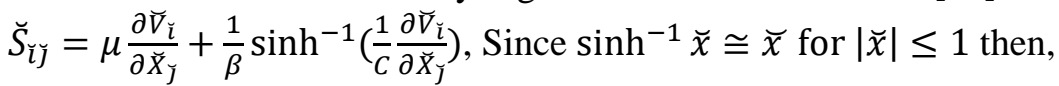

$$
\breve{S}_{\breve{l \jmath}}=\mu \frac{\partial \breve{V}_{\breve{l}}}{\partial \breve{X}_{\breve{\jmath}}}+\frac{1}{\beta}\left(\frac{1}{C} \frac{\partial \breve{V}_{\breve{l}}}{\partial \breve{X}_{\breve{\jmath}}}\right)
$$

where $\mu$ is the fluid dynamic viscosity, $\beta$ and $C$ are material constants, and $\breve{V}_{\breve{\imath}}=(\breve{u}, \breve{v}, \breve{w})$ and $\breve{X}_{\breve{J}}=$ $(\breve{x}, \breve{y}, \breve{z})$. Hence, the conditions are:

$$
\begin{gathered}
\breve{u}_{\breve{z}}=0, \breve{v}_{\breve{z}}=0, k^{\prime} \frac{\partial T}{\partial \breve{z}}=0 \text { at } \breve{z}=\breve{h}_{1} \\
\breve{u}=0, \breve{v}=0, k^{\prime} \frac{\partial T}{\partial \breve{z}}=-\breve{n}_{1}\left(T_{0}-T\right) \text { at } \breve{z}=\breve{h}_{2} \\
\bar{x}=\breve{x}-c \breve{t}, \bar{y}=\breve{y}, \bar{z}=\breve{z} \\
\bar{u}(\bar{x}, \bar{y}, \bar{z})=\breve{u}(\breve{x}, \breve{y}, \breve{z})-c, \bar{v}(\bar{x}, \bar{y}, \bar{z})=\breve{v}(\breve{x}, \breve{y}, \breve{z}), \bar{w}(\bar{x}, \bar{y}, \bar{z})=\breve{w}(\breve{x}, \breve{y}, \breve{z})
\end{gathered}
$$

in which $\breve{n}_{1}$ and $T_{0}$ indicate the heat transfer coefficient and temperature at walls, respectively. Then, by using the wave frame transformations and conditions (9-12), Eqs. (3)- (7) become:

$$
\begin{gathered}
\frac{\partial \bar{u}}{\partial \bar{x}}+\frac{\partial \bar{w}}{\partial \bar{z}}=0 \\
\rho\left[(\bar{u}+c) \frac{\partial \bar{u}}{\partial \bar{x}}+\bar{v} \frac{\partial \bar{u}}{\partial \bar{y}}+\bar{w} \frac{\partial \bar{u}}{\partial \bar{z}}-2 \bar{\Omega} \bar{v}\right]=-\frac{\partial \bar{P}}{\partial \bar{x}}+\frac{\partial \bar{S}_{\bar{x} \bar{x}}}{\partial \bar{x}}+\frac{\partial \bar{S}_{\bar{x} \bar{y}}}{\partial \bar{y}}+\frac{\partial \bar{S}_{\bar{x} \bar{z}}}{\partial \bar{z}}-\sigma \beta_{0}^{2}(\bar{u}+c) \\
\rho\left[(\bar{u}+c) \frac{\partial \bar{v}}{\partial \bar{x}}+\bar{v} \frac{\partial \bar{v}}{\partial \bar{y}}+\bar{w} \frac{\partial \bar{v}}{\partial \bar{z}}+2 \bar{\Omega}(\bar{u}+c)\right]=-\frac{\partial \bar{P}}{\partial \bar{y}}+\frac{\partial \bar{S}_{\bar{y} \bar{x}}}{\partial \bar{x}}+\frac{\partial \bar{S}_{\bar{y} \bar{y}}}{\partial \bar{y}}+\frac{\partial \bar{S}_{\bar{y} \bar{z}}}{\partial \bar{z}} \\
\rho\left[(\bar{u}+c) \frac{\partial \bar{w}}{\partial \bar{x}}+\bar{v} \frac{\partial \bar{w}}{\partial \bar{y}}+\bar{w} \frac{\partial \bar{w}}{\partial \bar{z}}\right]=-\frac{\partial \bar{P}}{\partial \bar{z}}+\frac{\partial \bar{S}_{\bar{z} \bar{x}}}{\partial \bar{x}}+\frac{\partial \bar{S}_{\bar{z} \bar{y}}}{\partial \bar{y}}+\frac{\partial \bar{S}_{\bar{z} \bar{z}}}{\partial \bar{z}} \\
\rho c_{p} \frac{d T}{d \bar{t}}=k^{\prime}\left[\frac{\partial^{2} T}{\partial \bar{x}^{2}}+\frac{\partial^{2} T}{\partial \bar{y}^{2}}+\frac{\partial^{2} T}{\partial \bar{z}^{2}}\right]+\bar{S}_{\bar{x} \bar{x}} \frac{\partial \bar{u}}{\partial \bar{x}}+\bar{S}_{\bar{x} \bar{z}}\left(\frac{\partial \bar{u}}{\partial \bar{z}}+\frac{\partial \bar{w}}{\partial \bar{x}}\right)+\bar{S}_{\bar{z} \bar{z}} \frac{\partial \bar{w}}{\partial \bar{z}}+Q_{0}\left(T-T_{O}\right)
\end{gathered}
$$

It is worth noting the definitions of the non-dimensional quantities [14], which are:

$$
\begin{aligned}
& x=\frac{\bar{x}}{\lambda}, y=\frac{\bar{y}}{\lambda}, z=\frac{\bar{z}}{d}, t=\frac{c \bar{t}}{\lambda}, P=\frac{d^{2} \bar{P}}{\mu \lambda c}, h_{1,2}=\frac{\bar{h}_{1,2}}{d} m=\frac{m^{\prime}}{d}, \phi=\frac{\phi^{\prime}}{d}, \varepsilon_{1}=\frac{a}{d}, \varepsilon_{2}=\frac{b}{d} \\
& u=\frac{\bar{u}}{c}, v=\frac{\bar{v}}{c}, w=\frac{\bar{w}}{c}, \theta=\frac{T-T_{0}}{T_{0}}, k^{\prime}=\frac{R}{d}, \delta=\frac{d}{\lambda}, d^{\prime}=\frac{d_{1}}{d}, v=\frac{\mu}{\rho}, M^{2}=\frac{\sigma \beta_{0}^{2} d^{2}}{\mu} \\
& \left.T^{\prime}=\frac{R e \bar{\Omega} d}{c}, R e=\frac{c d}{v}, B i=\frac{h d}{k^{\prime}}, \operatorname{Pr}=\frac{\mu c_{p}}{k^{\prime}}, S=\frac{Q_{0} d^{2}}{k^{\prime}}, \alpha=\frac{1}{\mu \beta C}, B r=\frac{c^{2} \mu}{k^{\prime} T^{\prime}}, S_{i j}=\frac{d \breve{S}_{\breve{l}}}{\mu c}\right)
\end{aligned}
$$

where the terms $R e, v, T^{\prime}, \alpha, B r, \operatorname{Pr}, S, \varepsilon_{1}, \varepsilon_{2}, B i$, and $M^{2}$ represent the Reynolds number, kinematic viscosity, Taylor number, fluid parameter, Brinkman number, Prandtl number, heat source/sink parameter, amplitude ratio parameters, Biot number, and Hartman number, respectively. Now, if we use Eq. (18) and the facts of $u=\psi_{z}, w=-\delta \psi_{x}$ into Eqs. (13)- (17), the following dimensionless equations are obtained:

$$
\begin{gathered}
\frac{\partial P}{\partial x}-\frac{\partial S_{x z}}{\partial z}-2 T^{\prime} v+M^{2}(u+1)=0 \\
\frac{\partial P}{\partial y}-\frac{\partial S_{y z}}{\partial z}+2 T^{\prime}\left(\frac{\partial \psi}{\partial Z}+1\right)=0 \\
\frac{\partial P}{\partial z}=0 \\
\frac{\partial^{2} \theta}{\partial z^{2}}+B r S_{x z} \psi_{z z}+S \theta=0
\end{gathered}
$$




$$
S_{x z}=(1+\alpha) \psi_{z z}, S_{y z}=(1+\alpha) v_{z}
$$

The dimensionless form of the boundary and boundary conditions are

$$
\begin{gathered}
h_{1}=1+m x+\varepsilon_{1} \sin 2 \pi x \\
h_{2}=-d^{\prime}-m x-\varepsilon_{2}(\sin 2 \pi x+\phi) \\
\psi=0, \psi_{z z}=0, v_{z}=0, \frac{\partial \theta}{\partial z}=0 \text { at } z=h_{1} \\
\psi=F_{1}, \psi_{z}=-1, v=0, \frac{\partial \theta}{\partial z}-B i \theta=0 \text { at } z=h_{2}
\end{gathered}
$$

The pressure $\Delta p$ and the flow rate $F_{2}$ are:

$$
\Delta P=\int_{0}^{1} \frac{\partial P}{\partial x} d x, F_{1}=\int_{h_{1}}^{h_{2}} \psi_{z} d z, F_{2}=\int_{h_{1}}^{h_{2}} v d z
$$

Eqs. (19) and (20) and the secondary flow pressure due to the rotational effect were neglected, and then we get:

\section{Solutions of the problem}

$$
\begin{gathered}
\frac{\partial}{\partial z}\left[\frac{\partial S_{x z}}{\partial z}+2 T^{\prime} v-M^{2}(u+1)\right]=0 \\
\frac{\partial S_{y z}}{\partial z}-2 T^{\prime}\left(\frac{\partial \psi}{\partial z}+1\right)=0
\end{gathered}
$$

This section reviews the results of velocities, temperature, and stream function analytically. Eqs. (22), (29), and (30) with the boundary condition equations (24) - (27) are solved analytically with the help of MATHEMATICA programming software. The solution is found in the form:

$$
\begin{aligned}
& \psi(z)=-z+\frac{C 5}{2 T^{\prime}}+e^{z k_{3}} c_{1}+e^{-z k_{3}} c_{2}+e^{z k_{4}} c_{3}+e^{-z k_{4}} c_{4} \\
& k_{3}=\sqrt{\frac{M^{2}-\sqrt{M^{4}-16\left(T^{\prime}\right)^{2}}}{2+2 \alpha}}, \quad k_{4}=\sqrt{\frac{M^{2}+\sqrt{M^{4}-16\left(T^{\prime}\right)^{2}}}{2+2 \alpha}}, k=\frac{2 T^{\prime}}{1+\alpha} \\
& v(z)=\frac{e^{-(k 3+k 4) z}\left(-2 c 4 e^{k 3 z} k k 3 T^{\prime}(1+\alpha)+e^{k 4 z}(A 2)\right)}{2 k 3 k 4 T^{\prime}(1+\alpha)}+c 6 \\
& A_{1}=\left(-2 \mathrm{c} 2 k T^{\prime}(1+\alpha)+2 \mathrm{c} 1 e^{2 \mathrm{k} 3 z} k T^{\prime}(1+\alpha)+\mathrm{c} 5 e^{\mathrm{k} 3 z} \mathrm{k} 3 z\left(k-2 T^{\prime}+k \alpha\right)\right) \\
& A_{2}=2 \mathrm{c} 3 e^{(\mathrm{k} 3+\mathrm{k} 4) z} k \mathrm{k} 3 T^{\prime}(1+\alpha)+\mathrm{k} 4\left(A_{1}\right) \\
& F_{2}=\left[\begin{array}{c}
\mathrm{c} 6(\mathrm{~h} 2-\mathrm{h} 1)+\frac{\mathrm{c} 4 k}{k_{4}{ }^{2}}\left(e^{-\mathrm{h} 2 \mathrm{k} 4}-e^{-\mathrm{h} 1 \mathrm{k} 4}\right) \\
-\frac{e^{-\mathrm{h} 1 \mathrm{k} 3} k\left(\mathrm{c} 3 e^{\mathrm{h} 1(\mathrm{k} 3+\mathrm{k} 4)} \mathrm{k} 3^{2}+\left(\mathrm{c} 2+\mathrm{c} 1 e^{2 \mathrm{~h} 1 \mathrm{k} 3}\right) \mathrm{k} 4^{2}\right)}{\mathrm{k} 3^{2} \mathrm{k} 4^{2}} \\
+\frac{e^{-\mathrm{h} 2 \mathrm{k} 3} k\left(\mathrm{c} 3 e^{\mathrm{h} 2(\mathrm{k} 3+\mathrm{k} 4)} \mathrm{k} 3^{2}+\left(\mathrm{c} 2+\mathrm{c} 1 e^{2 \mathrm{~h} 2 \mathrm{k} 3}\right) \mathrm{k} 4^{2}\right)}{\mathrm{k}^{2} \mathrm{k} 4^{2}} \\
+\frac{\mathrm{c} 5\left(k-2 T^{\prime}+k \alpha\right)}{4 T^{\prime}(1+\alpha)}\left(\mathrm{h} 2^{2}-\mathrm{h} 1^{2}\right)
\end{array}\right] \\
& \theta(z)=-B r e^{-2\left(k_{3}+k_{4}\right) z}\left[\begin{array}{c}
(1+\alpha)\left(\frac{\left(k 3^{2}-2 k 3 k 4+k 4^{2}+S\right)(N 6)}{(N 8)}\right. \\
+\frac{c 1^{2} e^{2(2 k 3+k 4) z} k 3^{4} S(N 5)}{(N 8)} \\
+\frac{2 c 3 e^{(k 3+2 k 4) z} k 4^{2}(N 4)(N 3)}{(N 8)} \\
+\frac{c 3^{2} e^{2(k 3+2 k 4) z} k 4^{4} S(N 2)}{(N 8)} \\
\left.+\frac{2 c 1 k 3^{2}\left(4 k 3^{2}+S\right)\left(4 k 4^{2}+S\right)(N 1)}{(N 8)}\right) \\
+\frac{e^{2(k 3+k 4) z} N 7(c 7 \operatorname{Cos}[\sqrt{S} z]+c 8 \operatorname{Sin}[\sqrt{S} z])}{(N 8)}
\end{array}\right]
\end{aligned}
$$


Where, $c i, i=1,2, \ldots, 8$ are constants which are found by using the boundary conditions below.

$$
\begin{aligned}
& N 1=\left(c_{2} e^{2\left(k_{3}+k_{4}\right) z}{k_{3}}^{2}\left({k_{3}}^{4}-2{k_{3}}^{2}\left({k_{4}}^{2}-S\right)+\left({k_{4}}^{2}+S\right)^{2}\right)\right. \\
& +e^{\left(3 k_{3}+k_{4}\right) z} k_{4}{ }^{2} S\left(c_{3} e^{2 k_{4} z}\left(k 3^{2}-2 k 3 k 4+k 4^{2}+S\right)\right. \\
& \left.\left.+c_{4}\left(k 3^{2}+2 k 3 k 4+k 4^{2}+S\right)\right)\right) \\
& N 2=\left(4{k_{3}}^{6}+S\left({k_{4}}^{2}+S\right)^{2}+{k_{3}}^{4}\left(-8{k_{4}}^{2}+9 S\right)+{k_{3}}^{2}\left(4 k_{4}{ }^{4}+6 k 4^{2} S+6 S^{2}\right)\right) \\
& N 3=\left(4 k_{3}{ }^{4}\left(4 k_{4}{ }^{2}+S\right)+8 k_{4} k_{3}{ }^{3}\left(4 k_{4}{ }^{2}+S\right)+2 S k_{3} k_{4}\left(4 k_{4}{ }^{2}+S\right)\right. \\
& +S\left(4 k_{4}^{4}+5 k_{4}^{2} S+S^{2}\right) \\
& \left.+k_{3}^{2}\left(16 k_{4}^{4}+24 k 4^{2} S+5 S^{2}\right)\right) \\
& N 4=\left(c_{2} e^{k_{4} z} k_{3}{ }^{2} S+c_{4} e^{k_{3} z} k_{4}{ }^{2}\left(k_{3}{ }^{2}-2 k_{3} k_{4}+k_{4}{ }^{2}+S\right)\right) \\
& N 5=\left({k_{3}}^{4}\left(4{k_{4}}^{2}+S\right)+\left({k_{4}}^{2}+S\right)^{2}\left(4{k_{4}}^{2}+S\right)+{k_{3}}^{2}\left(-8 k_{4}{ }^{4}+6{k_{4}}^{2} S+2 S^{2}\right)\right) \\
& N 6=\left(c_{4}^{2} e^{2 k_{3} z} k_{4}^{4}\left(4 k_{3}^{2}+S\right)\left(k_{3}^{2}+2 k_{3} k_{4}+k_{4}^{2}+S\right)\right. \\
& +2 c_{2} c_{4} e^{\left(k_{3}+k_{4}\right) z}{k_{3}}^{2}{k_{4}}^{2}\left(4{k_{3}}^{2}+S\right)\left(4{k_{4}}^{2}+S\right) \\
& \left.+c_{2}{ }^{2} e^{2 k_{4} z} k_{3}{ }^{4}\left(4 k_{4}{ }^{2}+S\right)\left(k_{3}{ }^{2}+2 k_{3} k_{4}+k_{4}{ }^{2}+S\right)\right) \\
& N 7=\left(4{k_{3}}^{2}+S\right)\left(4{k_{4}}^{2}+S\right)\left({k_{3}}^{4}-2{k_{3}}^{2}\left(4{k_{4}}^{2}+S\right)+\left(4 k_{4}{ }^{2}+S\right)^{2}\right) \\
& N 8=\left(( 2 k _ { 3 } - i \sqrt { S } ) ( k _ { 3 } - k _ { 4 } - i \sqrt { S } ) ( k _ { 3 } + k _ { 4 } - i \sqrt { S } ) ( 2 k _ { 3 } + i \sqrt { S } ) ( k _ { 3 } - k _ { 4 } + i \sqrt { S } ) \left(k_{3}+k_{4}\right.\right. \\
& \left.+i \sqrt{S})\left(-2 i k_{4}+\sqrt{S}\right)\left(2 i k_{4}+\sqrt{S}\right)\right) \text {. }
\end{aligned}
$$

\section{Results and Discussion}

We demonstarte Figures- 2- 21 to monitor the resulting effects when changing the parameter values on the axial $u$ and secondary $v$ velocities as well as $F_{2}$ against Hartmann parameter $(M)$, fluid parameter $(\alpha)$, Taylor number $\left(T^{\prime}\right)$, the amplitude parameters $\left(\varepsilon_{1}, \varepsilon_{2}\right)$, phase differences $(\phi)$, Biot number $(B i)$, heat source/sink parameter $(S)$, and Brinkman number $(B r)$.

\subsection{The Axial Velocity Distribution $u$}

Figure- 2 shows that $u$ is enhanced in the left side of the channel, but it is decayed in its right side when $T^{\prime}$ increases. The same outcomes are observed for the fluid parameter $\alpha$, as noted in Figure- 3 . One can observe from Figure- 4 that when $M$ increased, this will lead to the emergence of a force called "Lorentz force" due to the influence of the magnetic field exerted on the flow of the fluid. Thus, a resistance against the flow will be created, resulting in a diminishing $u$ in the left side, which has an increasing value in the right side. In Figure- 5, we can notice that we get the same results as in the previous figure for $m$. 


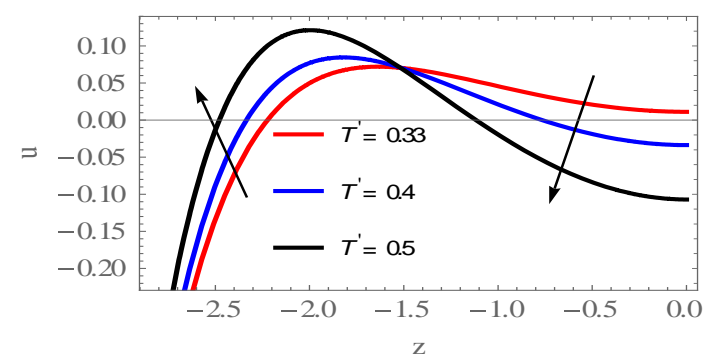

Figure 2- The axial velocity's diversity for $T^{\prime}$ when

$\alpha=0.05, M=4, m=0.07, \phi=\frac{3 \pi}{4}, d^{\prime}=3, \varepsilon_{1}=$ $1.5, \varepsilon_{2}=0.5, F_{1}=0.2, x=0.1$.

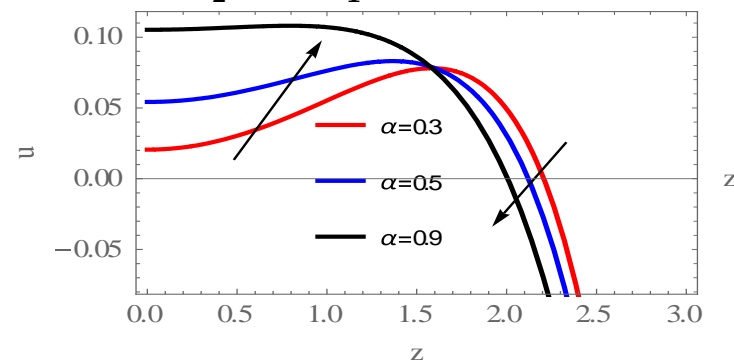

Figure 3- The axial velocity's diversity for $\alpha$ when

$$
\begin{gathered}
T^{\prime}=0.4, M=4, m=0.07, \phi=\frac{3 \pi}{4}, d^{\prime}=3, \varepsilon_{1}= \\
1.5, \varepsilon_{2}=0.5, F_{1}=0.2, x=0.1 .
\end{gathered}
$$

\subsection{The Secondary Velocity Distribution $v$}

Figure- 6 shows that if $T^{\prime}$ is increased, it leads to a decrease in velocity at the fluid flow center. To know the effect of $\alpha$ on the secondary velocity $v$, we notice that increasing the values of $\alpha$ leads to an increase in $v$. Figure- 7 illustrates this effect, for the same reasons of the effect of $\alpha$ on axial velocity $u$. Figure- 8 shows the effect of $M$ on $v$. One can notice that $v$ increases slowly by increasing the values of $M$. The influences of $m$ and $\phi$ parameters on the velocity $v$ are portrayed respectively in Figure- 9 and Figure-10. It is seen that $v$ decreases with the increase of $m$ and $\phi$. In Fig. 11, one can observe the effect of $F_{1}$ on the secondary velocity $v$, which increases in the channel.

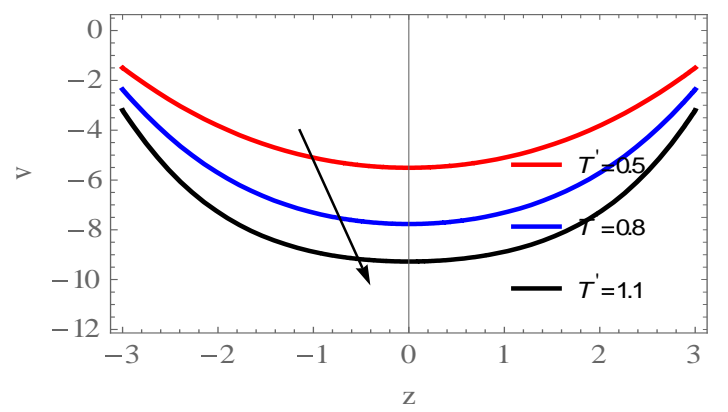

Figure 6-The secondary velocity's diversity for $T^{\prime}$ when $\alpha=0.05, M=5, m=0.07, \phi=\frac{3 \pi}{4}$,

$$
\begin{gathered}
d^{\prime}=3, \\
\varepsilon_{1}=1.5, \varepsilon_{2}=0.5, F_{1}=0.2, x=2 .
\end{gathered}
$$

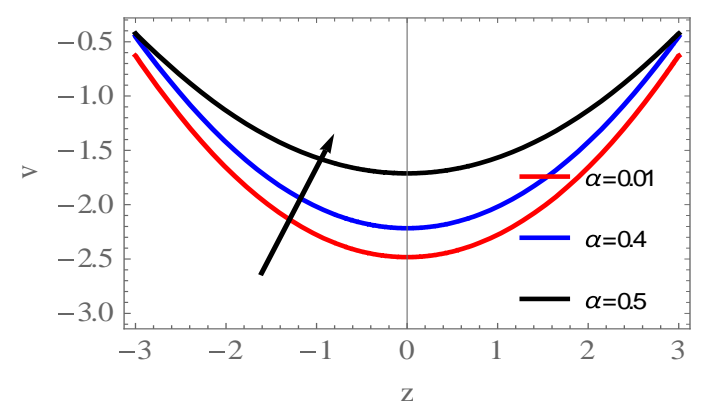

Figure 7- The secondary velocity's diversity for $\alpha$ when $T^{\prime}=0.2, M=5, m=0.07, \phi=\frac{3 \pi}{4}, d^{\prime}$ $=3$, $\varepsilon_{1}=1.5, \varepsilon_{2}=0.5, F_{1}=0.2, x=2$. 


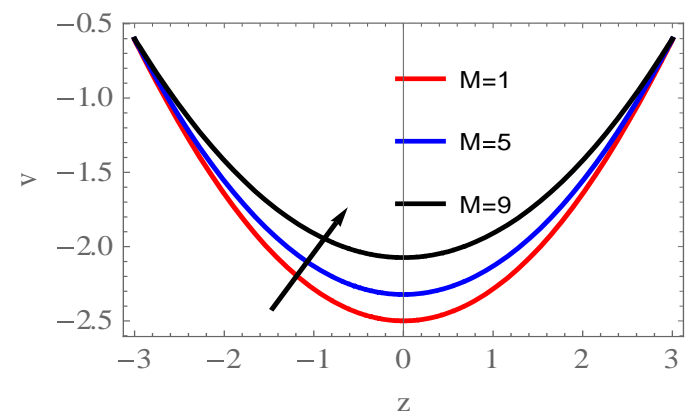

Figure 8- The secondary velocity's diversity for $M$ when $T^{\prime}=0.2, \alpha=0.05, m=0.07, \phi=$ $\frac{3 \pi}{4}$

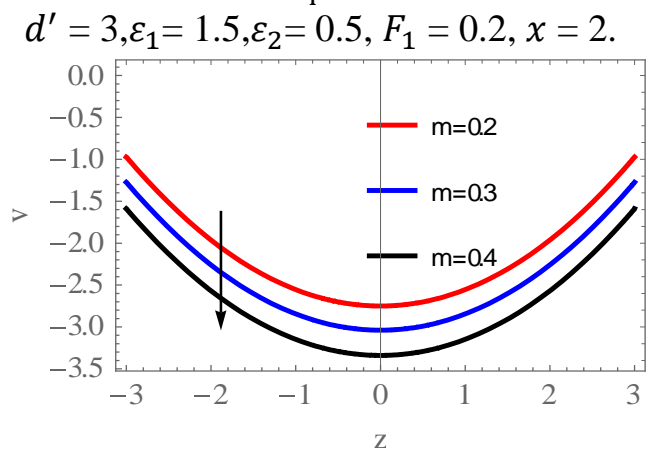

Figure 9 -The secondary velocity's diversity for $m$ when $T^{\prime}=0.2, \alpha=0.05, M=5, \phi=\frac{3 \pi}{4}$, $d^{\prime}=3$,

$$
\varepsilon_{1}=1.5, \varepsilon_{2}=0.5, F_{1}=0.2, x=2 .
$$

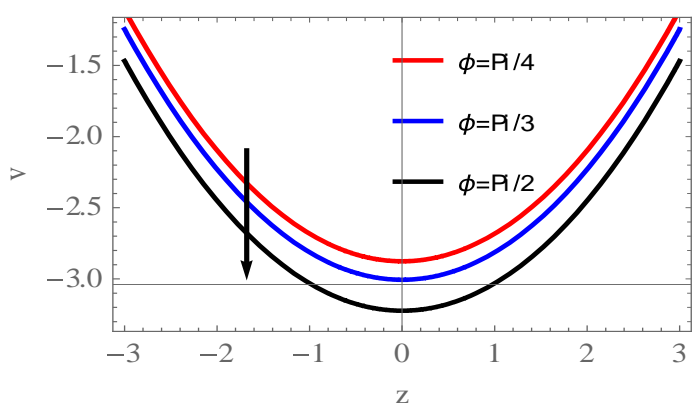

Figure 10 -The secondary velocity's diversity for $\phi$ when $T^{\prime}=0.2, \alpha=0.05, m=0.3, M=$

$$
5, d^{\prime}=3 \text {, }
$$

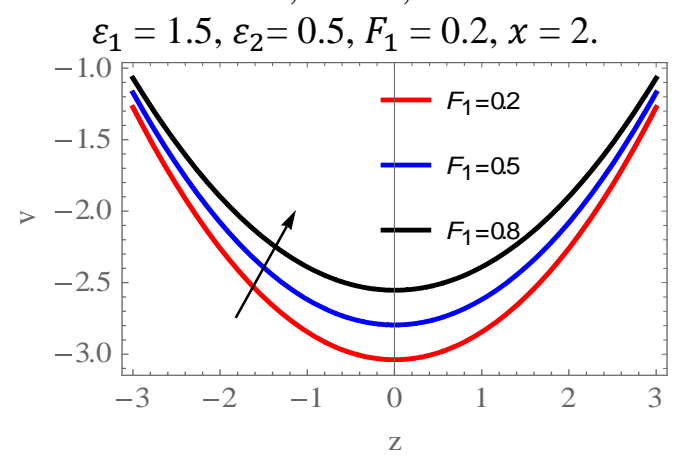

Figure 11 -The secondary velocity's diversity for $F_{1}$ when $T^{\prime}=0.2, \alpha=0.05, m=0.3$, $M=5, \phi=\frac{\pi}{4}, d^{\prime}=3 \varepsilon_{1}=1.5, \varepsilon_{2}=0.5, x=2$.

\subsection{The flow rate distribution}

Now, figures 12- 15 are drawn to show the variance in dimensionless flow rate due to the secondary velocity $F_{2}$ for some $\alpha$ and $T^{\prime}$, when $F_{1}=-1,-0.5$. In Figures- 12 and 13, we notice a decrease in $\left(F_{2}\right)$ if we increase , while in figures- 14 and 15, we observe an opposite results of $F_{2}$ if we increase some values of $T^{\prime}$.

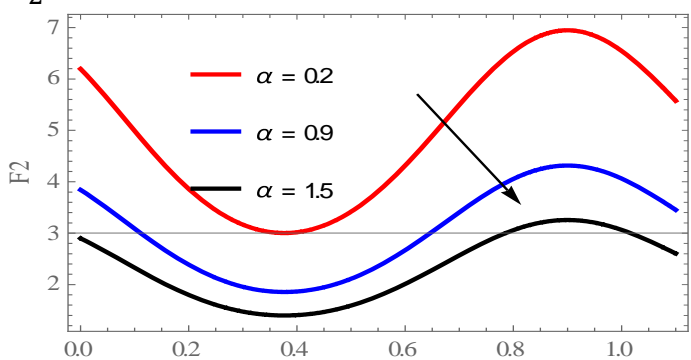

Figure -12 Diversity in $F_{2}$ for $\alpha$ when $F_{1}=-1$, $T^{\prime}=0.2, m=0.07, M=5, \phi=\frac{3 \pi}{4}, d^{\prime}=3$,

$$
\varepsilon_{1}=1.5, \varepsilon_{2}=0.5 \text {. }
$$

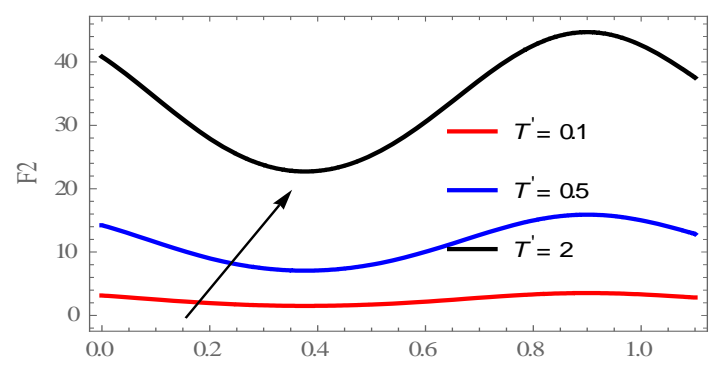

Figure -13 Diversity in $F_{2}$ for $\alpha$ when $F_{1}=$ 0.5 , $T^{\prime}=0.2, m=0.07, M=5, \phi=\frac{3 \pi}{4}, d^{\prime}=3$, $\varepsilon_{1}=1.5, \varepsilon_{2}=0.5$. 


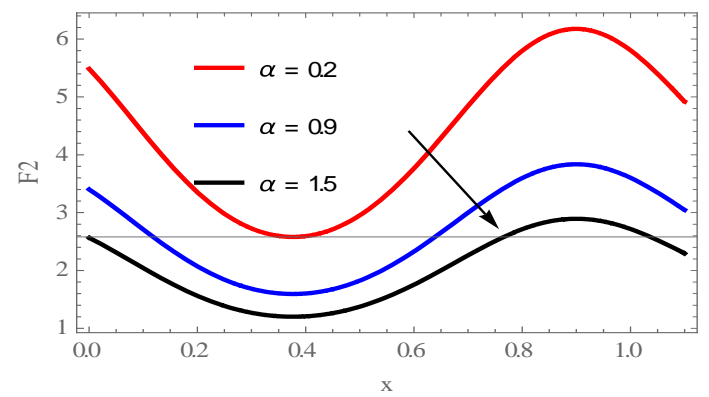

Figure -14 Diversity in $F_{2}$ for $T^{\prime}$ when $F_{1}=$ 1 ,

$$
\begin{gathered}
\alpha=0.2, m=0.07, M=5, \phi=\frac{3 \pi}{4}, d^{\prime}=3, \\
\varepsilon_{1}=1.5, \varepsilon_{2}=0.5 .
\end{gathered}
$$

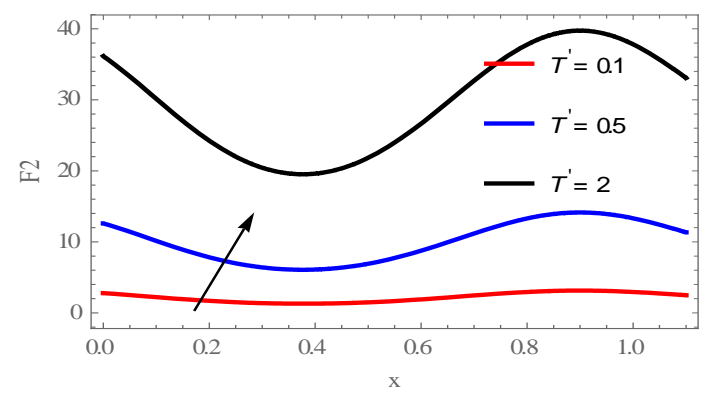

Figure -15 Diversity in $F_{2}$ for $T^{\prime}$ when $F_{1}=$ 0.5 ,

$$
\alpha=0.2, m=0.07, M=5, \phi=\frac{3 \pi}{4}, d^{\prime}=3,
$$$$
\varepsilon_{1}=1.5, \varepsilon_{2}=0.5 \text {. }
$$

\subsection{Temperature profile}

Now, temperature $(\theta)$ for different variables is demonstrated through Figures- 16-21. In Figure- 16, we observe, for fluid parameter $\alpha$ against $\theta$, that temperature distribution increases when the fluid parameter increases. In Figure- 17, we observe that if $T^{\prime}$ increases then $\theta$ also increases. If both of $S$ and $B i$ increase, respectively, we notice no effect on temperature (Figures- 18 and 19). Figure- 20 shows that if we increase $B r, \theta$ decays. From Figure- 21, we notice that if $M$ is enhanced, the temperature gradually decreases. The following figures are drawn for the values of $d^{\prime}=3, T^{\prime}=0.4, m$ $=0.07, M=4, \phi=\frac{3 \pi}{4}, \varepsilon_{1}=1.5, \alpha=0.05, \varepsilon_{2}=0.5, F_{1}=0.2, x=0.1, B i=2, S=0.2$, and $B r=0.2$.

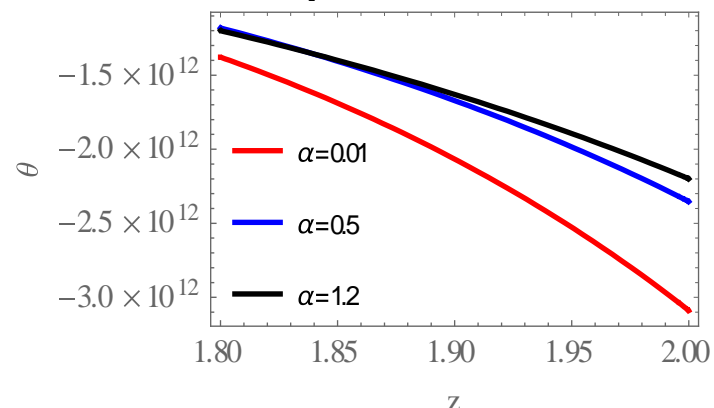

Figure16-Diversity in $\theta$ for $\alpha=\{0.01,0.5,1.2\}$

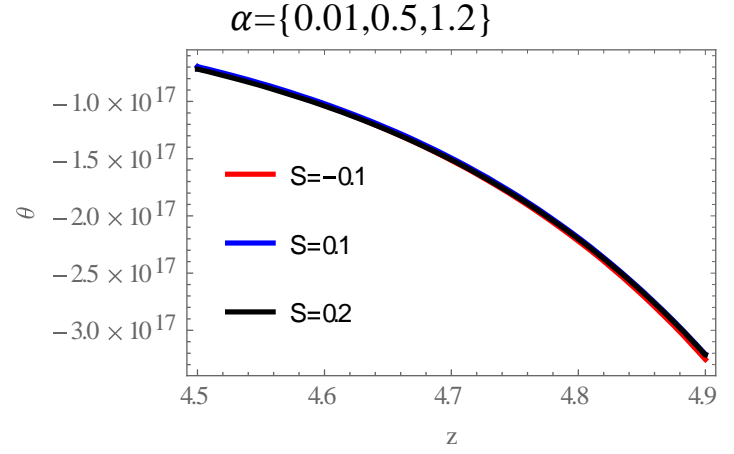

Figure 18 -Diversity in $\theta$ for $S=\{$ $0.1,0.1,0.2\}$

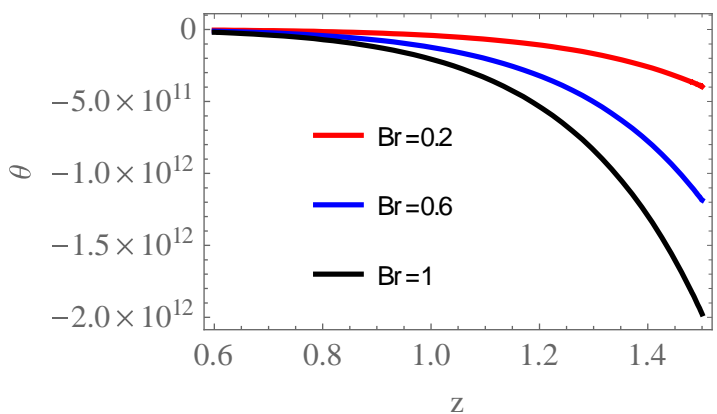

Figure 20-Diversity in $\theta$ for $B r=\{0.2$, $0.6,1\}$

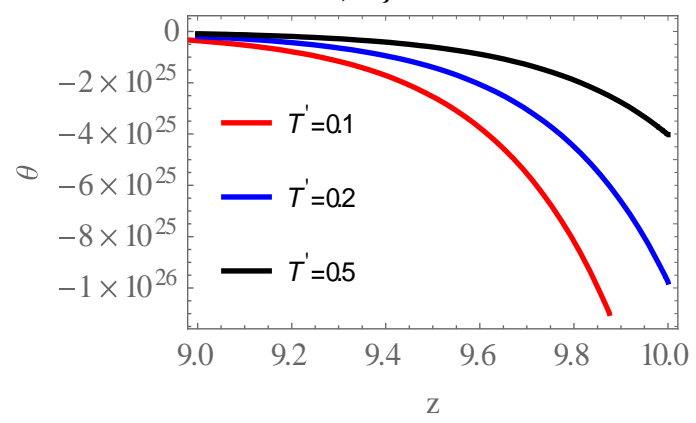

Figure 17-Diversity in $\theta$ for $T^{\prime}=\{0.1,0.2,0.5\}$ 


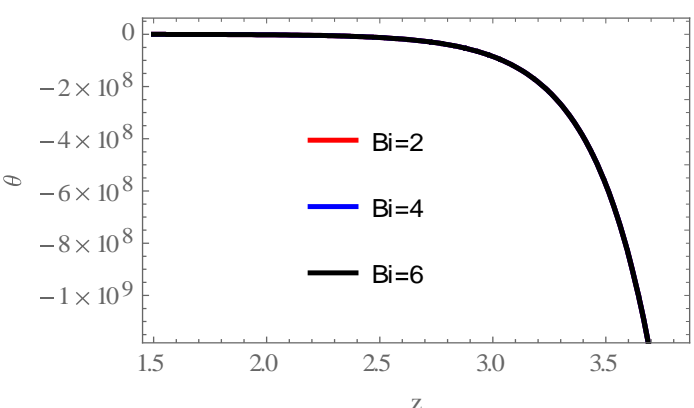

Figure -19 Diversity in $\theta$ for $B i=\{2,4,6\}$

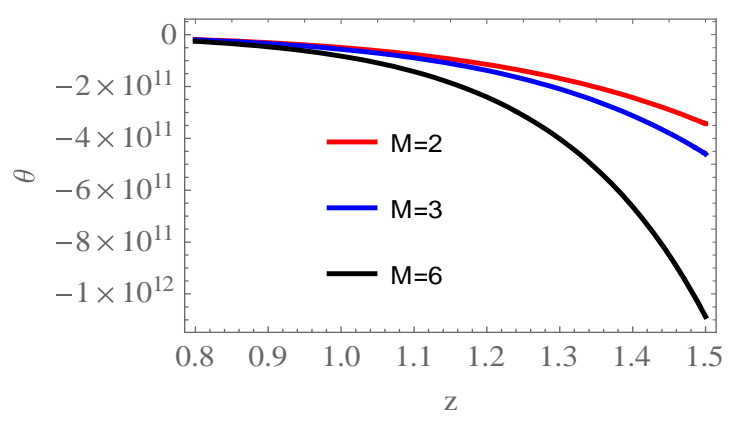

Figure -21 Diversity in $\theta$ for $M=\{2,3,6\}$

\subsection{Trapping Phenomenon}

The next interesting part of this article is trapping. This fascinating phenomenon, normally known as bolus trapping phenomenon, occurs in the peristaltic flows, where the closed stream lines entice the quantity of fluid which is internal to the channel/tube and close to the walls. This trapping bolus moves within the course of the wave propagation. The behavior of stream function is illustrated in Figures- 22-30. One can observe in Figure- 22 that the value of $T^{\prime}$ increments at that point when the value of increases. It can be delineated from Figure- 23 that if $\alpha$ is enhanced the value of bolus decreases gradually. One can notice from Figure- 24 that the size of the trapped bolus is reduced in the channel upon increasing the magnetic parameter $M$. Figure- 25 shows that there is an increase in trapping when $m$ increases. Decreasing behavior in the size of the trapped bolus is seen upon rising up the amplitude $\varepsilon_{2}$ (Figure- 26). Figure- 27 shows that as $\phi$ increases, the bolus decreases. Figure-28 shows that as $d^{\prime}$ increase the bolus tends to rise. The impact of $\varepsilon_{1}$ is illustrated in figure 29 , which shows that as $\varepsilon_{1}$ increases, the bolus increases. While Figure- 30 shows that upon increasing the values of $F_{1}$, bolus size tends to rise.

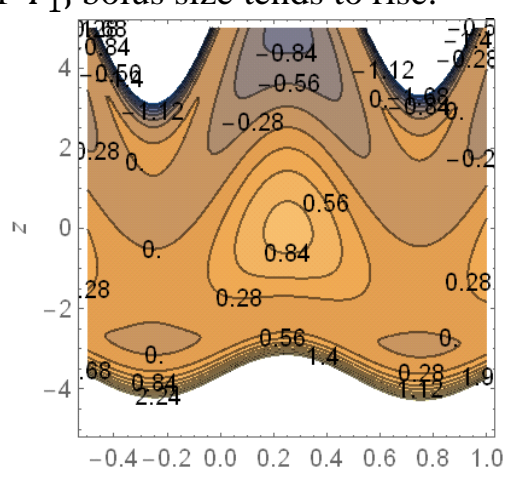

a

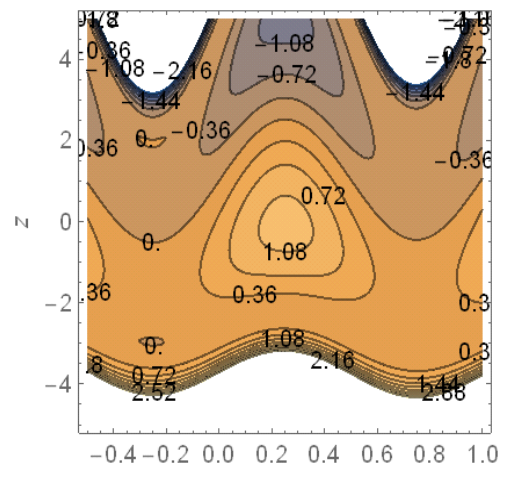

$\mathrm{b}$

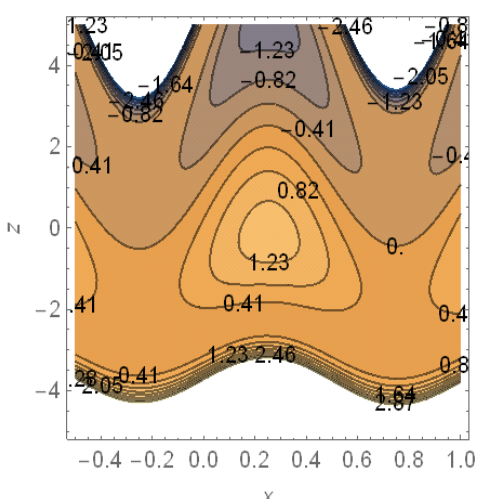

$x$

Figure 22- Effects of (a) $T^{\prime}=0.5$, (b) $T^{\prime}=0.6$, (c) $T^{\prime}=0.68$ on the stream lines $\psi$ when $\alpha=0.05, M=3$ $, m=0.07, \phi=\pi, d^{\prime}=3, \varepsilon_{1}=1.5, \varepsilon_{2}=0.5, F_{1}=0.2$.

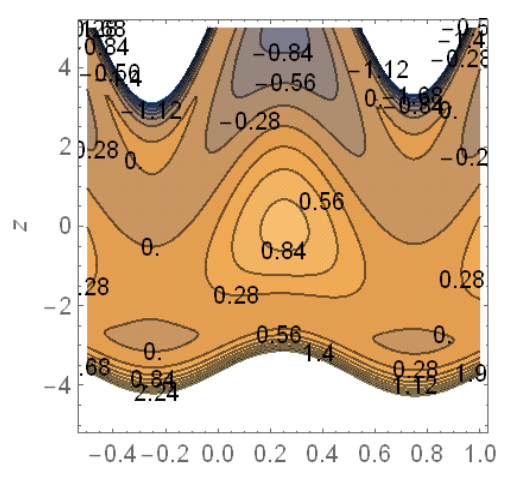

a

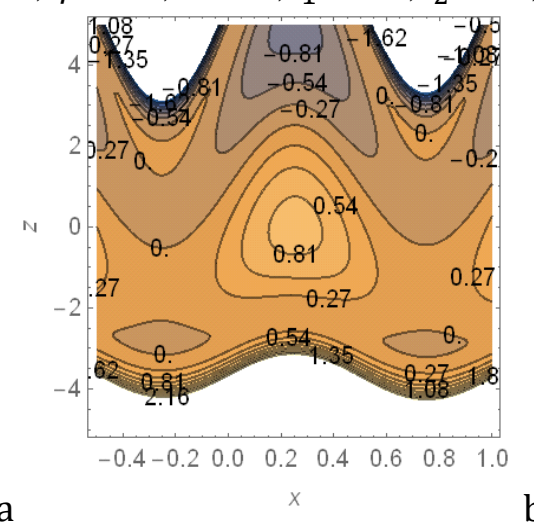

$\mathrm{b}$

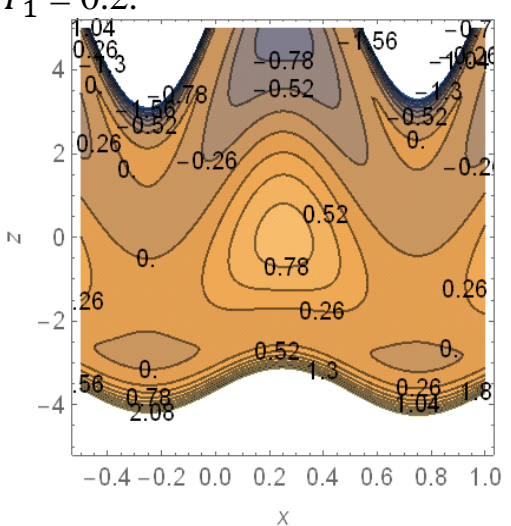

$x$

Figure 23-Effects of (a) $\alpha=0.05$, (b) $\alpha=0.06$, (c) $\alpha=0.08$ on the stream lines $\psi$ when $T^{\prime}=0.5, M=3$ $, m=0.07, \phi=\pi, d^{\prime}=3, \varepsilon_{1}=1.5, \varepsilon_{2}=0.5, F_{1}=0.2$. 

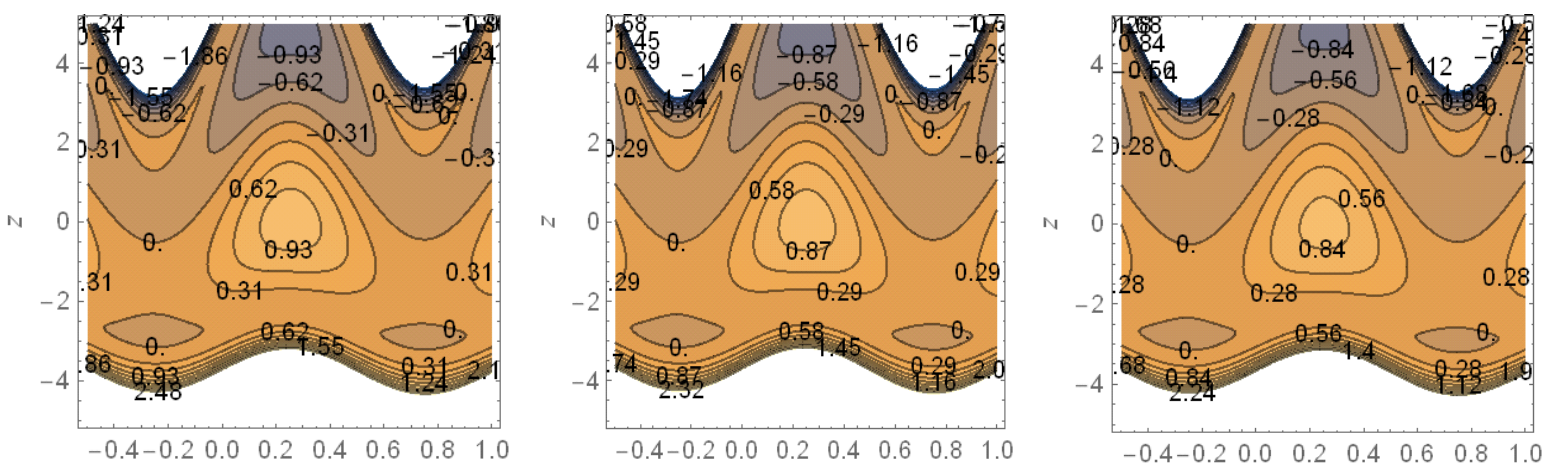

a

b

c

Figure 24 -Effects of (a) $M=2.1$, (b) $M=2.5$, (c) $M=3$ on the stream lines $\psi$ when $\alpha=0.05, T^{\prime}=0.5$,
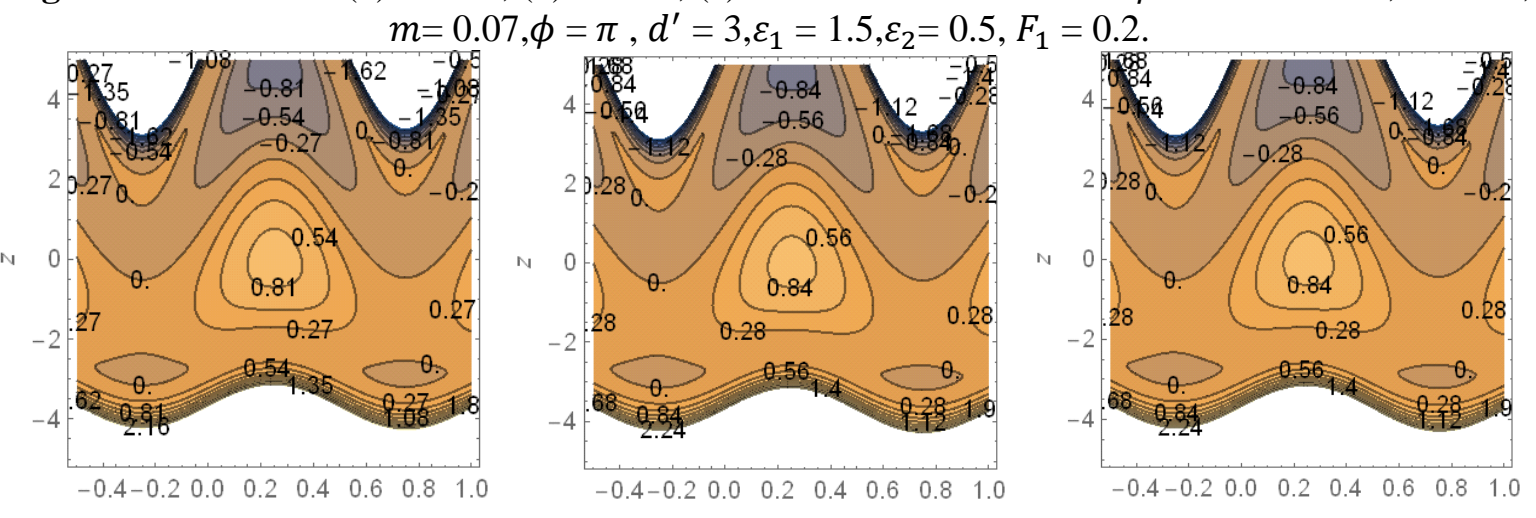

a

Figure 25- Effects of (a) $m=0.05$, (b) $m=0.07$, (c) $m=0.09$ on the stream lines $\psi$ when $\alpha=0.05, T^{\prime}=$ 0.5 ,
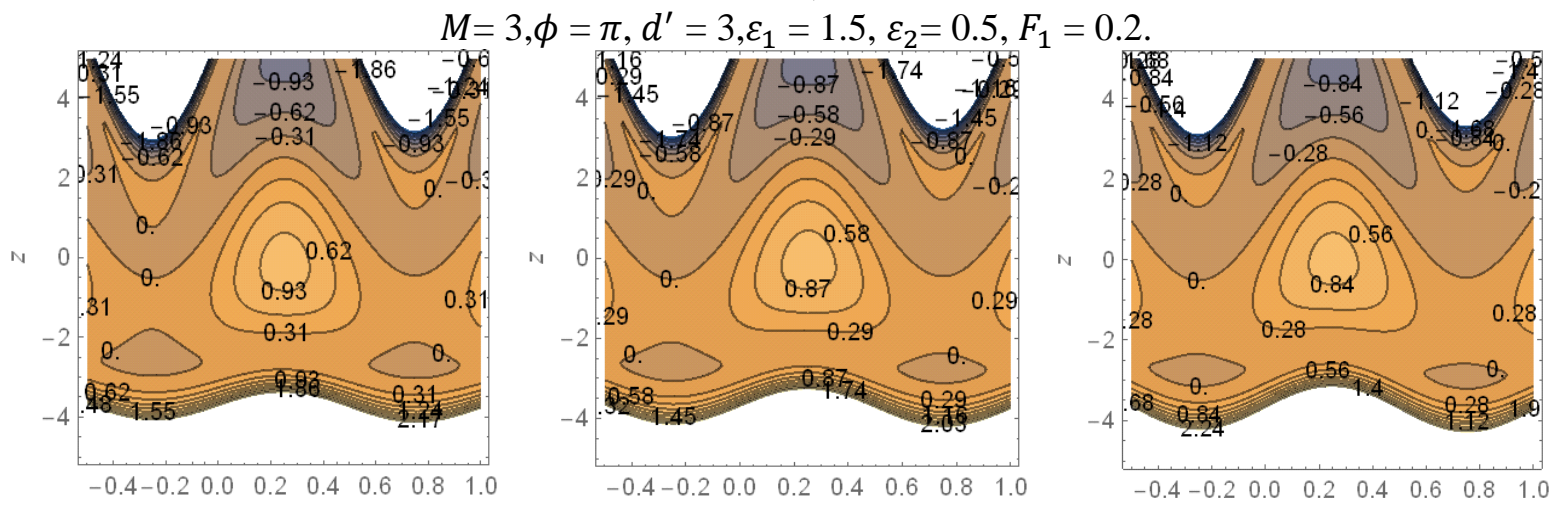

a

b

Figure 26- Effects of (a) $\varepsilon_{2}=0.3$, (b) $\varepsilon_{2}=0.4$, (c) $\varepsilon_{2}=0.5$ on the stream lines $\psi$ when $\alpha=0.05, T^{\prime}=0.5$,
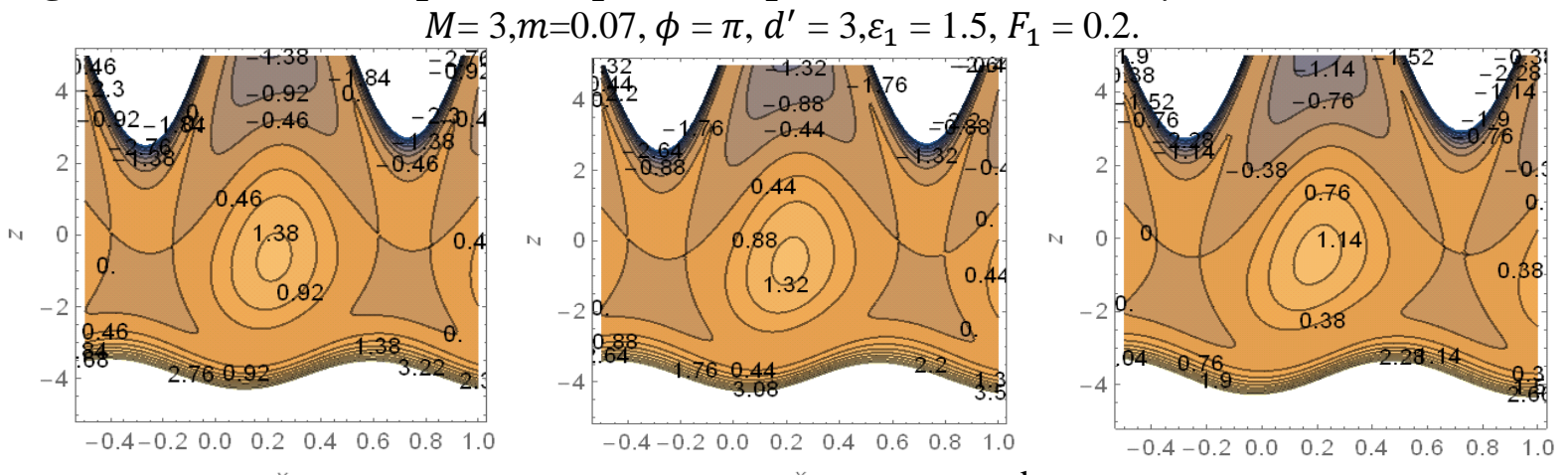

Figure 27-Effects of (a) $\phi=\frac{\pi}{4}$, (b) $\phi=\frac{\pi}{3}$, (c) $\phi=\frac{\pi}{2}$ on the stream lines $\psi$ when $\alpha=0.05, T^{\prime}=0.5$, $M=3, m=0.07, d^{\prime}=3, \varepsilon_{1}=1.5, \varepsilon_{2}=0.5, F_{1}=0.2$. 

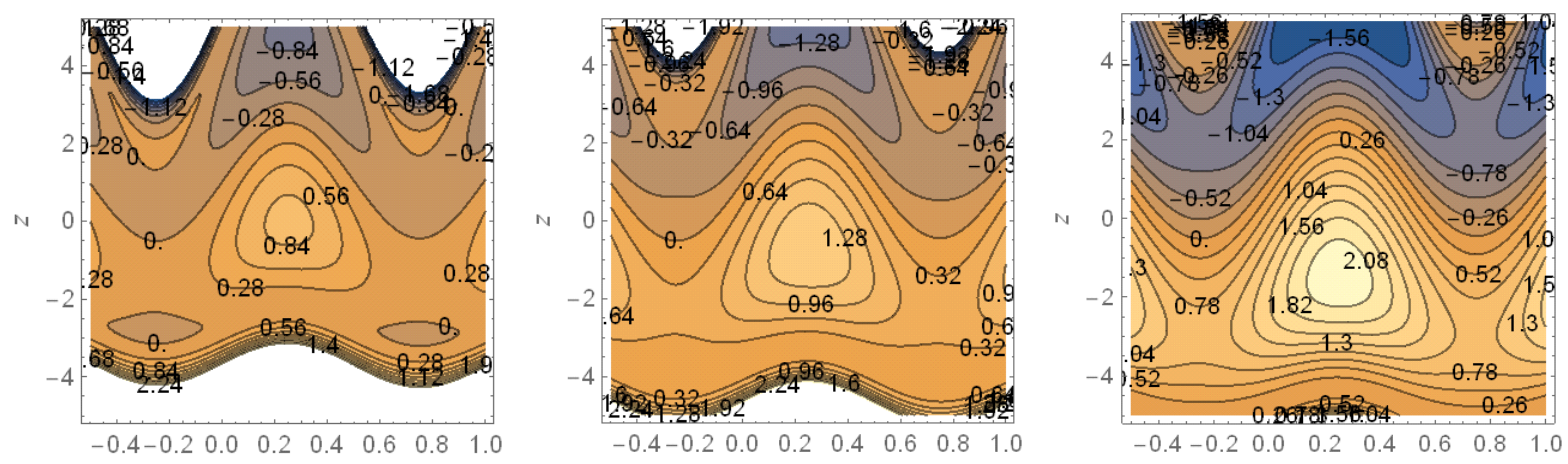

a

b

Figure 28-Effects of (a) $d^{\prime}=3$, (b) $d^{\prime}=4$, (c) $d^{\prime}=5$ on the stream lines $\psi$ when $\alpha=0.05, T^{\prime}=0.5$, $M=3, m=0.07, \phi=\pi, \varepsilon_{1}=1.5, \varepsilon_{2}=0.5, F_{1}=0.2$.

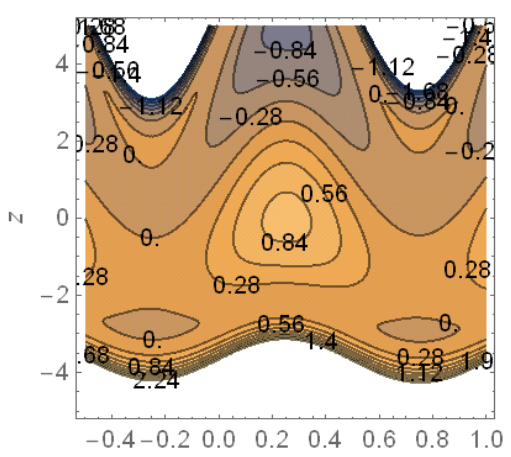

a

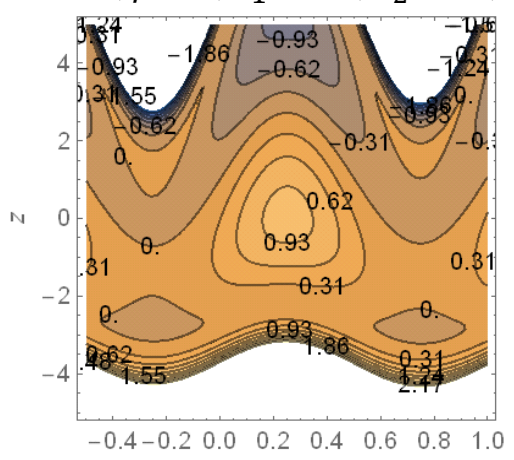

b

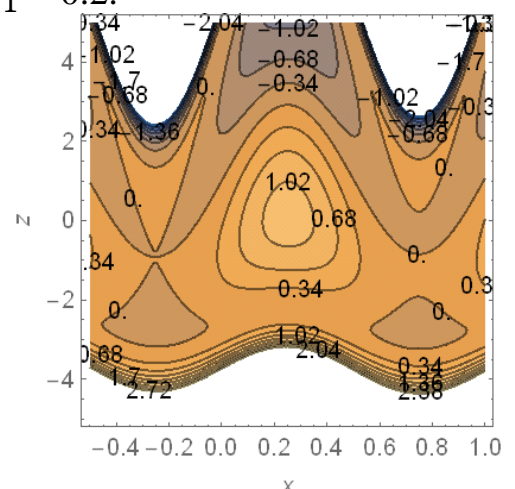

c

Figure 29-Effects of (a) $\varepsilon_{1}=1.5$, (b) $\varepsilon_{1}=1.7$, (c) $\varepsilon_{1}=1.9$ on the stream lines $\psi$ when $\alpha=0.05, T^{\prime}=0.5$, $M=3, m=0.07, \phi=\pi, d^{\prime}=3, \varepsilon_{2}=0.5, F_{1}=0.2$.

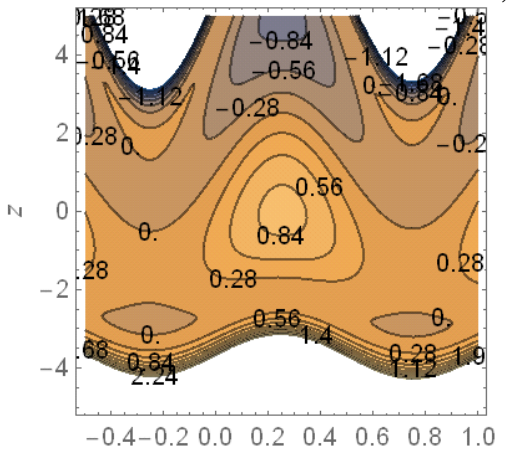

a

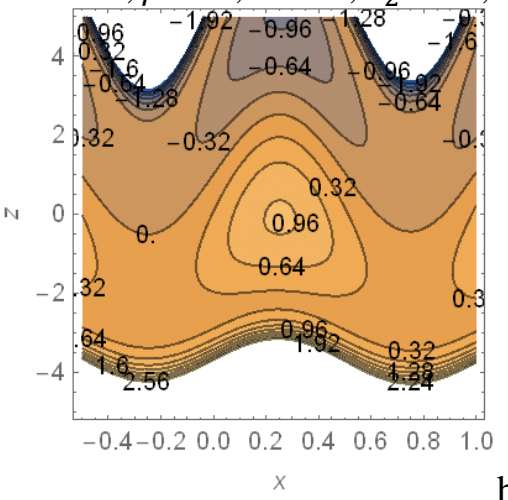

$\mathrm{b}$

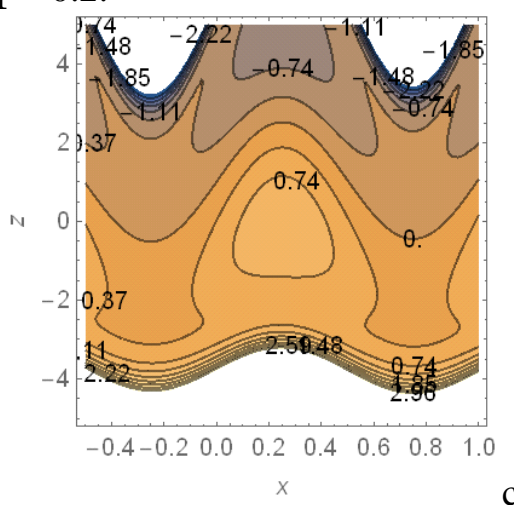

Figure 30-Effects of (a) $F_{1}=0.2$, (b) $F_{1}=0.4$, (c) $F_{1}=0.6$ on the stream lines $\psi$ when $\alpha=0.05, T^{\prime}=0.5$, $M=3, m=0.07, \phi=\pi, d^{\prime}=3, \varepsilon_{1}=1.5, \varepsilon_{2}=0.5$.

\section{Conclusions}

In this paper, the heat transfer and the magnetic field were discussed and the equations that describe the motion of flow for Ree-Eyring fluid were simplified under assumptions of low Reynolds number and long wavelength. These equations were solved analytically; the main results of the flow problem are summarized as follows.

When increasing $M$, the velocity $u$ increases in one side of the fluid course, while it decreases at the other; we also obtain the same results when increasing the amount of $m$, but when $M$ increases the velocity $v$ increases, while it decreases with the increase in $m$. As for the temperature it decreases due to the increase in the size of the magnetic field, but there is no effect on it profile for both $B i$ and $S$. For $T^{\prime}$ and $\alpha$, when $T^{\prime}$ increases, $u$ increases in one side and decreases in the other. $\alpha$ and $v$ increase with increasing $\alpha$, but $v$ decreases with increasing $T^{\prime}$. However, opposite results are obtained with respect to $F_{2}$. The temperature increases with increasing $\alpha$ and $T^{\prime}$. As for the 
phenomenon of ingestion that occurs in the fluid stream, it increases with increasing the values of $d^{\prime}, \varepsilon_{1}, T^{\prime}, m$, and $F_{1}$, while it decreases with increasing $\phi, \alpha, \varepsilon_{2}$, and $M$.

\section{References}

1. H. M. Shawky.2009." Pulsatile flow with heat transfer of dusty magnetohydrodynamic Ree-Eyring fluid through a channel," Heat Mass Transfer, 45:1261-1269.

2. N. S. Akbar, S. Nadeem. 2012." Characteristics of heating scheme and mass transfer on the peristaltic flow for an Eyring-Powell fluid in an endoscope," Int. J. Heat Mass Transfer, 55(1): 375383.

3. R. Ellahi, M. M. Bhatti, A. A. Khan. 2015."Mathematical analysis of peristaltic transport of an Eyring- Powell fluid through a porous rectangular duct," J. Wulfenia, 22(1): 266-283.

4. T. Hayat, H. Zahir, A. Alsaedi. B. Ahmad. 2017." Heat Transfer Analysis on Peristaltic Transport of Ree- Eyring Fluid in Rotating Frame”, Chinese Journal of Physics, 55:1894-1907.

5. S. R. Ridha, A. M. Abdulhadi. 2013."Unsteady heat transfer analysis on the MHD flow of a second grade fluid in a channel with porous medium," Iraqi journal of science, 54(1):174181.

6. F. A. Adnan, A. M. Abdulhadi. 2019."Effect of an inclined magnetic field on peristaltic flow of Bingham plastic fluid in an inclined symmetric channel with slip conditions," Iraqi Journal of Science, 60(7): 1551- 1574.

7. M. I. Khan, S. A. Khan, T. Hayat, M. F. Javed and A. Alsaedi. 2019.“Entropy generation in radiative flow of Ree-Eyring fluid due to due rotating disks," International Journal of Numerical Methods for Heat \& Fluid Flow, 29(6): 2057-2079.

8. W. S. Khudair, D. G. S. Al-Khafajy. 2018." Influence of heat transfer on Magneto hydrodynamics oscillatory flow for williamson fluid through a porous medium", Iraqi Journal of Science, 59(IB): 389-397.

9. T. Hayat, R. Ellahi, F.M. Mahomed. 2009.'The analytical solutions for magnetohydrodynamic flow of a third order fluid in a porous medium", Z. Naturforsch. A, 64 (9- 10): 531- 539.

10. A. M. Abd-Alla, S. M. Abo-Dahab, R. D. Al-Simery. 2013." Effect of rotation on peristaltic flow of a micropolar fluid through a porous medium with an external magnetic field," J. Magn. Magn. Mater. 348: 33-43.

11. A. Khalid, I. Khan, A. Khan, S. Shafie.2015. "Unsteady MHD free convection flow of Casson fluid past over an oscillating vertical plate embedded in a porous medium," Eng. Sci. Technol. Int. J., 18(3): 309-317.

12. K. S. Mekheimer, A. M. Salem, A. Z. Zaher. 2014."Peristatcally induced MHD slip flow in a porous medium due to a surface acoustic wavy wall", Journal of the Egyptian Mathematical Society. 22(1): 143-151.

13. M. Hatami D. Jing Majeed A. Yousif. 2018." Three-Dimensional Analysis of Condensation Nanofluid Film on an Inclined Rotating Disk by Efficient Analytical Methods," Arab Journal of Basic and Applied Sciences, 25(1): 28-73.

14. R. E. Powell, H. Eyrin. 1944." Mechanism for relaxation theory of viscosity," Nature, 154(55): $427-428,1944$. 